\title{
High Facial Distereoselectivity in Intra- and Intermolecular Reactions of Chiral Benzylic Cations
}

\author{
Friedrich Mühlthau, Oliver Schuster ${ }^{\ddagger}$ and Thorsten Bach ${ }^{*}$
}

Lehrstuhl für Organische Chemie I, Technische Universität München, Lichtenbergstr. 4, 85747 Garching, Germany

\section{Supporting Information}

\section{Synthesis of the Test Systems 1-3}

Compound 1a was synthesized from 2-methyl-1,4-diphenylbutan-1-one according to the following reference: Hornback, J. M.; Proehl, G. S. J. Am. Chem. Soc. 1979, 101, 7367-7373.

Compound $\mathbf{1 b}$ was synthesized from 3-phenylpropanal and $\alpha$-bromostyrene according to the following references: Anderson, S. C.; Smith, J. C.; Swarbrick, M. E. J. Chem. Soc., Perkin Trans. 1, 1997, 1517-1522; Miyaoka, H.; Shida, H.; Yamada, N.; Mitome, H.; Yamada, Y. Tetrahedron Lett. 2002, 43, 2227-2230.

Compound 1c was synthesized from 3-phenylpropanal and $\alpha$-bromostyrene similar as compound 1b and further transformed according to the following reference: Mulzer, J.; Funk, G. Synthesis 1995, 101-112.

Compound 2 was synthesized according to the following references: Tamura, Y.; Bayomi, S. M.; Sumoto, K.; Ikeda, M. Synthesis 1977, 693-694; Satoh, T.; Kobayashi, S.; Nakanishi, S.; Horiguchi, K.; Irisa, S. Tetrahedron 1999, 55, 2515-2528.

Compound 3 was synthesized from 2-methyl-1,4-diphenylbutan-1-one according to the following reference: Groenewold, G. S.; Chess, E. K.; Gross, M. L. J. Am. Chem. Soc. 1984, 106, 539-542.

\footnotetext{
‡ Lehrstuhl für Anorganische und Analytische Chemie, Technische Universität München, Lichtenbergstr. 4, 85747 Garching, Germany

* thorsten.bach@ch.tum.de
} 


\section{Intramolecular Reactions}

Representative procedure for the cyclization reaction

$59.1 \mathrm{mg}(250 \mu \mathrm{mol})$ of styrene 1a were dissolved in dry dichloromethane under an argon atmosphere. $2.5 \mathrm{~mL}$ of a $1 \mathrm{M}$ solution of trifluoro methane sulfonic acid in $\mathrm{F}_{2} \mathrm{ClCCFCl}_{2}$ were added at room temperature. The reaction was stopped by adding $20 \mathrm{~mL}$ saturated aqueous $\mathrm{NaHCO}_{3}$ after one minute at room temperature. The reaction mixture was diluted with $20 \mathrm{~mL}$ of diethyl ether. After washing the organic layer with $10 \mathrm{~mL}$ of saturated aqueous $\mathrm{NaHCO}_{3}, 20 \mathrm{~mL}$ of saturated aqueous $\mathrm{NaCl}$, and drying with $\mathrm{NaSO}_{4}$, concentration in vacuo yielded $56.0 \mathrm{mg}$ $(95 \%)$ of a colourless oil. The diastereomeric ratio of the cyclization product $4 \mathbf{a}$ was trans/cis = $98 / 2$.

Spectroscopic Data of trans-1,2,3,4-tetrahydro-1,2-dimethyl-1-phenylnaphthalene (4a)<smiles>CC1CCc2ccccc2C1(C)c1ccccc1</smiles>

$\boldsymbol{R}_{\mathbf{f}} 0.27$ (pentane)

${ }^{1}$ H-NMR (500 MHz, CDCl $): \delta 0.79\left(\mathrm{~d},{ }^{3} J=6.8 \mathrm{~Hz}, 3 \mathrm{H}\right), 1.52(\mathrm{~s}, 3 \mathrm{H}), 1.71-1.79(\mathrm{~m}, 2 \mathrm{H})$, 2.12-2.22 (m, $1 \mathrm{H}), 2.89\left(\mathrm{dt},{ }^{2} \mathrm{~J}=16.4 \mathrm{~Hz},{ }^{3} \mathrm{~J}=4.6 \mathrm{~Hz}, 1 \mathrm{H}\right), 2.94-3.04(\mathrm{~m}, 1 \mathrm{H}), 6.71\left(\mathrm{~d},{ }^{3} \mathrm{~J}=\right.$ $7.2 \mathrm{~Hz}, 1 \mathrm{H}), 6.98\left(\mathrm{t},{ }^{3} \mathrm{~J}=7.2 \mathrm{~Hz}, 1 \mathrm{H}\right), 7.03-7.11$ (m, $\left.2 \mathrm{H}\right), 7.12-7.21(\mathrm{~m}, 3 \mathrm{H}), 7.22-7.28$ (m, $2 \mathrm{H})$.

${ }^{13}$ C-NMR (90 MHz, $\mathrm{CDCl}_{3}$ ): $\delta 16.4$ (q), 22.9 (q), 27.5 (t), 29.9 (t), 41.4 (d), 47.1 (d), 125.5 (d), 125.6 (d), 125.8 (d), 127.7 (d), 128.3 (d), 128.8 (d), 130.3 (d), 136.3 (s), 146.7 (s), 151.0 (s). 
Spectroscopic Data of trans-2-chloro-1,2,3,4-tetrahydro-1-methyl-1-phenylnaphthalene (4b)

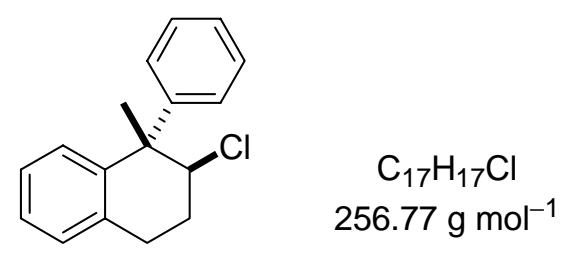

$\boldsymbol{R}_{\mathbf{f}} 0.20$ (pentane)

${ }^{1}$ H-NMR (500 MHz, $\mathrm{CDCl}_{3}$ ): $\delta 1.85$ (s, $\left.3 \mathrm{H}\right), 2.14-2.25$ (m, $2 \mathrm{H}$ ), $2.99\left(\mathrm{dt},{ }^{2} J=17.2 \mathrm{~Hz},{ }^{3} \mathrm{~J}=\right.$ $7.1 \mathrm{~Hz}, 1 \mathrm{H}), 3.14\left(\mathrm{dt},{ }^{2} J=17.2 \mathrm{~Hz},{ }^{3} \mathrm{~J}=6.3 \mathrm{~Hz}, 1 \mathrm{H}\right), 4.60\left(\mathrm{dd},{ }^{3} \mathrm{~J}=7.3 \mathrm{~Hz},{ }^{3} \mathrm{~J}=4.5 \mathrm{~Hz}, 1 \mathrm{H}\right)$, $6.93\left(\mathrm{~d},{ }^{3} \mathrm{~J}=7.9 \mathrm{~Hz}, 1 \mathrm{H}\right), 7.09-7.19(\mathrm{~m}, 5 \mathrm{H}), 7.21\left(\mathrm{t},{ }^{3} \mathrm{~J}=7.6 \mathrm{~Hz}, 1 \mathrm{H}\right), 7.28\left(\mathrm{t},{ }^{3} \mathrm{~J}=7.6 \mathrm{~Hz}\right.$, $2 \mathrm{H})$.

${ }^{13}$ C-NMR (90 MHz, $\mathrm{CDCl}_{3}$ ): $\delta 25.6(\mathrm{q}), 27.8$ (t), 28.6 (t), 48.7 (s), 69.6 (d), 126.4 (d), 126.5 (d), 126.6 (d), 128.0 (d), 128.1 (d), 128.7 (d), 129.6 (d), 134.8 (s), 142.7 (s), 148.6 (s).

Spectroscopic Data of 2-(trans-1,2,3,4-tetrahydro-1-methyl-1-phenylnaphthalen-2-yl)isoindoline1,3-dione (4c)

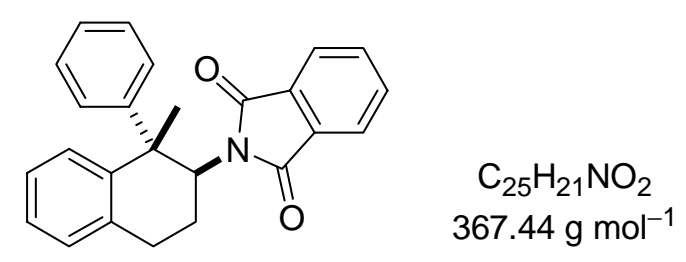

$\boldsymbol{R}_{\mathbf{f}} 0.20$ (pentane)

${ }^{1}$ H-NMR (360 MHz, $\mathrm{CDCl}_{3}$ ): $\delta 1.78$ (s, $\left.3 \mathrm{H}\right), 1.94-2.06$ (m, $\left.1 \mathrm{H}\right), 3.04-3.33$ (m, $\left.3 \mathrm{H}\right), 4.83$ (dd, $\left.{ }^{3} J=12.4 \mathrm{~Hz},{ }^{3} J=2.8 \mathrm{~Hz}, 1 \mathrm{H}\right), 6.77\left(\mathrm{~d},{ }^{3} \mathrm{~J}=7.9 \mathrm{~Hz}, 1 \mathrm{H}\right), 7.02-7.09(\mathrm{~m}, 1 \mathrm{H}), 7.11-7.25$ (m, H 7), 7.57-7.89 (m, 4 H).

${ }^{13}$ C-NMR (90 MHz, $\mathrm{CDCl}_{3}$ ): $\delta 24.4$ (t), 25.3 (q), 31.0 (t), 50.5 (s), 60.2 (d), 123.3 (d), 126.1 (d), 126.2 (d), 126.5 (d), 127.8 (d), 128.6 (d), 128.7 (d), 131.4 (s), 132.2 (s), 133.9 (d), 135.3 (s), 145.7 (s), 147.9 (s), two aromatic signals are superimposed. 
Spectroscopic Data of trans-1,2,3,4-tetrahydro-1-methyl-1-phenylnaphthalen-2-ol (4d) ${ }^{\dagger}$

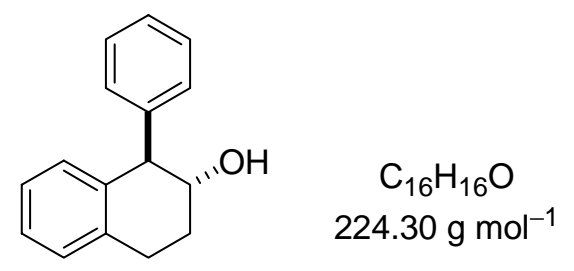

${ }^{1}$ H-NMR (500 MHz, CDCl $): \delta 1.55$ (bs, $1 \mathrm{H}$ ), 1.93 (dtd, ${ }^{2} J=12.7 \mathrm{~Hz},{ }^{3} J=9.7 \mathrm{~Hz},{ }^{3} J=6.8 \mathrm{~Hz}$, $1 \mathrm{H}), 2.17-2.24(\mathrm{~m}, 1 \mathrm{H}), 2.97-3.09(\mathrm{~m}, 2 \mathrm{H}), 3.98\left(\mathrm{~d},{ }^{3} J=8.2 \mathrm{~Hz}, 1 \mathrm{H}\right), 4.08$ (ddd, ${ }^{3} J=9.8 \mathrm{~Hz}$, $\left.{ }^{3} \mathrm{~J}=8.2 \mathrm{~Hz},{ }^{3} \mathrm{~J}=3.2 \mathrm{~Hz}, 1 \mathrm{H}\right), 6.75\left(\mathrm{~d},{ }^{3} \mathrm{~J}=7.7 \mathrm{~Hz}, 1 \mathrm{H}\right), 7.01-7.06(\mathrm{~m}, 1 \mathrm{H}), 7.11-7.17(\mathrm{~m}, 2 \mathrm{H})$, 7.17-7.20 (m, 2 H), 7.26-7.30 (m, 1 H), 7.32-7.37 (m, 2 H).

${ }^{13}$ C-NMR (90 MHz, $\mathrm{CDCl}_{3}$ ): $\delta 27.7$ (t), 29.5 (t), 55.3 (d), 73.8 (d), 126.0 (d), 126.2 (d), 126.9 (d), 128.4 (d), 128.7 (d), 129.6 (d), 130.2 (d), 136.1 (s), 137.8 (s), 143.3 (s).

Spectroscopic Data of trans-1,2,3,4-tetrahydro-2-methyl-1-phenylnaphthalene $(\mathbf{4 e})^{\ddagger}$

$\boldsymbol{R}_{\mathbf{f}} 0.36$ (pentane)

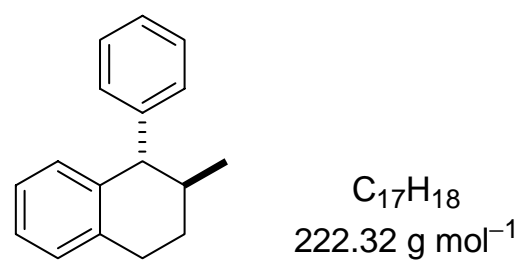

${ }^{1} \mathbf{H}-\mathbf{N M R}\left(500 \mathrm{MHz}, \mathrm{CDCl}_{3}\right): \delta 0.95\left(\mathrm{~d},{ }^{3} J=6.5 \mathrm{~Hz}, 3 \mathrm{H}\right), 1.60\left(\mathrm{dtd},{ }^{2} J=13.4 \mathrm{~Hz},{ }^{3} J=11.3 \mathrm{~Hz}\right.$, $\left.{ }^{3} J=5.1 \mathrm{~Hz}, 1 \mathrm{H}\right), 1.95-2.04(\mathrm{~m}, 2 \mathrm{H}), 2.90\left(\mathrm{dt},{ }^{2} J=16.6 \mathrm{~Hz},{ }^{3} J=4.3 \mathrm{~Hz}, 1 \mathrm{H}\right), 3.02$ (ddd, $\left.{ }^{2} J=16.6 \mathrm{~Hz},{ }^{3} J=11.5 \mathrm{~Hz},{ }^{3} J=5.1 \mathrm{~Hz}, 1 \mathrm{H}\right), 3.60\left(\mathrm{~d},{ }^{3} J=9.3 \mathrm{~Hz}, 1 \mathrm{H}\right), 6.72\left(\mathrm{~d},{ }^{3} J=7.9 \mathrm{~Hz}\right.$, $1 \mathrm{H}), 7.00\left(\mathrm{t},{ }^{3} \mathrm{~J}=7.2 \mathrm{~Hz}, 1 \mathrm{H}\right), 7.07-7.17(\mathrm{~m}, 4 \mathrm{H}), 7.23\left(\mathrm{t},{ }^{3} \mathrm{~J}=7.4 \mathrm{~Hz}, 1 \mathrm{H}\right), 7.30\left(\mathrm{t},{ }^{3} \mathrm{~J}=7.4 \mathrm{~Hz}\right.$, $2 \mathrm{H})$.

${ }^{13}$ C-NMR (90 MHz, CDCl $): \delta 20.5$ (q), 29.4 (t), 30.7 (t), 37.4 (d), 54.2 (d), 125.5 (d), 125.6 (d), 126.0 (d), 128.2 (d), 128.6 (d), 129.4 (d), 130.3 (d), 137.0 (s), 140.0 (s), 146.4 (s).

$(\dagger)$ Laus, G.; Tourwé, D.; Van Binst, G. Heterocycles 1984, 22, 311-331.

(†) Tournier, H.; Longeray, R.; Dreux, J. Bull. Soc.Chim. Fr. 1972, 3214-3221. 


\section{Intermolecular Reaction}

Representative procedure for the intermolecular reaction

A solution of $96.2 \mathrm{mg}(500 \mu \mathrm{mol})$ of the alcohol $5 \mathrm{in} 10 \mathrm{~mL}$ of dry dichloromethane was cooled to $-78{ }^{\circ} \mathrm{C}$. After addition of $102 \mathrm{mg}(630 \mu \mathrm{mol}) \mathrm{HBF}_{4} \cdot \mathrm{OEt}_{2}$ and stirring for 5 minutes, $136 \mathrm{mg}$ $(2.00 \mathrm{mmol})$ of furan were added and the solution was warmed to ambient temperature over 15 minutes. The workup was conducted the same way as described for the intramolecular case. $114 \mathrm{mg}(94 \%)$ of product 6a were obtained as a yellowish oil in a diastereomeric ratio of syn/anti $=98 / 2$.

Spectroscopic Data of 2-(syn-2,3,3-trimethyl-1-phenylbutyl)furan (6a)<smiles>C[C@H]([C@H](O)[18OH])[C@@H](c1ccccc1)C(C)(C)C</smiles>

$\boldsymbol{R}_{\mathbf{f}} 0.19$ (pentane)

${ }^{1} \mathbf{H}-\mathbf{N M R}\left(\mathrm{CDCl}_{3}, 360 \mathrm{MHz}\right): \delta 0.83(\mathrm{~s}, 9 \mathrm{H}), 0.94\left(\mathrm{~d},{ }^{3} \mathrm{~J}=7.2 \mathrm{~Hz}, 3 \mathrm{H}\right), 1.93\left(\mathrm{qd},{ }^{3} J=7.2 \mathrm{~Hz},{ }^{3} J\right.$ $=4.4 \mathrm{~Hz}, 1 \mathrm{H}), 4.39\left(\mathrm{~d},{ }^{3} J=4.4 \mathrm{~Hz}, 1 \mathrm{H}\right), 6.08\left(\mathrm{~d},{ }^{3} \mathrm{~J}=3.1 \mathrm{~Hz}, 1 \mathrm{H}\right), 6.30\left(\mathrm{dd},{ }^{3} J=3.1 \mathrm{~Hz},{ }^{3} J=\right.$ $1.5 \mathrm{~Hz}, 1 \mathrm{H}), 7.13-7.30$ (m, $5 \mathrm{H}), 7.37$ (d, ${ }^{3} \mathrm{~J}=1.5 \mathrm{~Hz}, 1 \mathrm{H}$ );

${ }^{13}$ C-NMR (CDCl, $90 \mathrm{MHz}$ ): $\delta 11.5$ (q), 28.2 (q), 34.2 (s), 45.9 (d), 49.5 (d), 107.9 (d), 110.3 (d), 126.0 (d), 128.3 (d), 140.9 (d), 144.6 (s), 156.5 (s), two aromatic signals are superimposed. 
Spectroscopic Data of 1-(syn-1-(2,4-dimethoxyphenyl)-2,3,3-trimethylbutyl)benzene (6b)

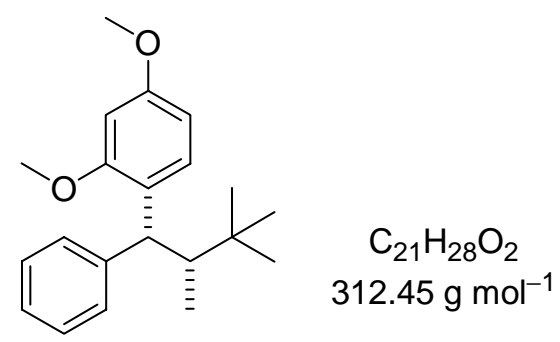

$\boldsymbol{R}_{\mathbf{f}} 0.30$ (pentane/ $\left.\mathrm{Et}_{2} \mathrm{O}=95 / 5\right)$

${ }^{1}$ H-NMR (500 MHz, $\mathrm{CDCl}_{3}$ ): $\delta 0.79\left(\mathrm{~d},{ }^{3} \mathrm{~J}=6.9 \mathrm{~Hz}, 3 \mathrm{H}\right), 0.84$ (s, $\left.9 \mathrm{H}\right), 2.32-2.42(\mathrm{~m}, 1 \mathrm{H}), 3.77$ (s, $3 \mathrm{H}), 3.81$ (s, $3 \mathrm{H}), 4.52-4.64(\mathrm{~m}, 1 \mathrm{H}), 6.40-6.46$ (m, $2 \mathrm{H}), 7.08-7.16(\mathrm{~m}, 2 \mathrm{H}), 7.23\left(\mathrm{t},{ }^{3} \mathrm{~J}=\right.$ $7.6 \mathrm{~Hz}, 2 \mathrm{H}), 7.37$ (d, $\left.{ }^{3} \mathrm{~J}=7.6 \mathrm{~Hz}, 2 \mathrm{H}\right)$.

${ }^{13}$ C-NMR (90.6 MHz, $\mathrm{CDCl}_{3}$ ): $\delta 14.4$ (q), 29.0 (q), 34.8 (s), 45.4 (d), 55.3, 55.5, 98.5 (d), 104.2 (d), 125.3 (d), 126.7 (s), 128.1 (d), 128.8 (d), 130.3 (d), 147.5 (s), 157.8 (s), 158.5 (s), one aliphatic signal is superimposed.

Spectroscopic Data of 2-(syn-2,3,3-trimethyl-1-phenylbutyl)-1-tosyl-1H-pyrrole (6c)

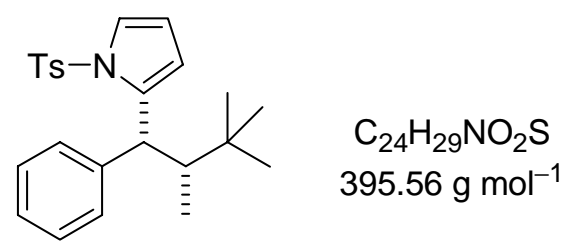

$\boldsymbol{R}_{\mathbf{f}} 0.34$ (pentane $/ \mathrm{Et}_{2} \mathrm{O}=9 / 1$ )

${ }^{1}$ H-NMR (360 MHz, $\mathrm{CDCl}_{3}$ ): $\delta 0.81\left(\mathrm{~d},{ }^{3} J=7.2 \mathrm{~Hz}, 3 \mathrm{H}\right), 0.83$ (s, $\left.9 \mathrm{H}\right), 1.84-1.94(\mathrm{~m}, 1 \mathrm{H}), 2.24$ (s, $3 \mathrm{H}), 5.02\left(\mathrm{~d},{ }^{3} \mathrm{~J}=5.0 \mathrm{~Hz}, 1 \mathrm{H}\right), 6.27\left(\mathrm{t},{ }^{3} \mathrm{~J}=3.5 \mathrm{~Hz}, 1 \mathrm{H}\right), 6.37-6.41$ (m, $\left.1 \mathrm{H}\right), 6.84-6.96$ (m, $4 \mathrm{H}), 6.99-7.06$ (m, $3 \mathrm{H}), 7.24\left(\mathrm{~d},{ }^{3} J=8.4 \mathrm{~Hz}, 2 \mathrm{H}\right), 7.34-7.39(\mathrm{~m}, 1 \mathrm{H})$.

${ }^{13}$ C-NMR (90 MHz, $\mathrm{CDCl}_{3}$ ): $\delta 12.0$ (q), 21.6 (q), 28.7 (q), 34.5 (s), 42.4 (d), 51.6 (d), 111.4 (d), 115.2 (d), 122.6 (d), 125.3 (d), 126.8 (d), 127.9 (d), 128.7 (d), 129.4 (d), 136.0 (s), 136.1 (s), 144.1 (s), 144.3 (s). 
Spectroscopic Data of 2-(syn-Methyl-5-2,3,3-trimethyl-1-phenylbutyl)-thiophene (6d)

$\boldsymbol{R}_{\mathbf{f}} 0.26$ (pentane)

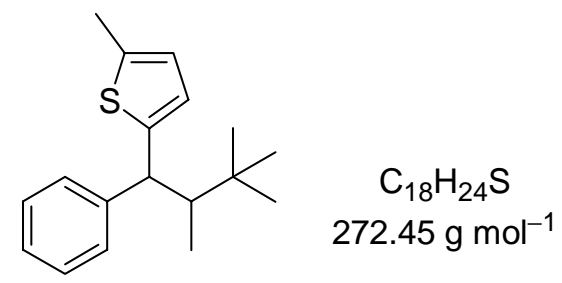

${ }^{1}$ H-NMR (500 MHz, $\mathrm{CDCl}_{3}$ ): $\delta 0.87$ (s, $\left.9 \mathrm{H}\right), 0.99\left(\mathrm{~d},{ }^{3} \mathrm{~J}=7.1 \mathrm{~Hz}, 3 \mathrm{H}\right), 2.02-2.10(\mathrm{~m}, 1 \mathrm{H}), 2.43$ (s, $3 \mathrm{H}), 4.45\left(\mathrm{~d},{ }^{3} \mathrm{~J}=5.0 \mathrm{~Hz}, 1 \mathrm{H}\right), 6.54-6.59(\mathrm{~m}, 1 \mathrm{H}), 6.65-6.68(\mathrm{~m}, 1 \mathrm{H}), 7.17\left(\mathrm{t},{ }^{3} \mathrm{~J}=7.2 \mathrm{~Hz}\right.$, $1 \mathrm{H}), 7.28\left(\mathrm{t},{ }^{3} \mathrm{~J}=7.6 \mathrm{~Hz}, 2 \mathrm{H}\right), 7.35\left(\mathrm{~d},{ }^{3} \mathrm{~J}=7.7 \mathrm{~Hz}, 2 \mathrm{H}\right)$.

${ }^{13}$ C-NMR (90.6 MHz, $\mathrm{CDCl}_{3}$ ): $\delta 12.7$ (q), 15.4 (q), 28.6 (q), 34.6 (s), 48.8 (d), 49.0 (d), 124.4 (d), 125.8 (d), 125.9 (d), 128.2 (d), 128.3 (d), 138.1 (s), 144.5 (s), 146.5 (s).

Spectroscopic Data of syn-4,5,5-trimethyl-1,3-diphenylhexan-1-one (10)<smiles>C[C@@H](C(C)(C)C)C(C)(C)[C@@H](CC(=O)c1ccccc1)c1ccccc1</smiles>

$\boldsymbol{R}_{\mathbf{f}} 0.24$ (pentane/ $\mathrm{Et}_{2} \mathrm{O}=95 / 5$ )

${ }^{1}$ H-NMR (360 MHz, $\mathrm{CDCl}_{3}$ ): $\delta$ 0.98-1.04 (m, $\left.12 \mathrm{H}\right), 1.62$ (qd, ${ }^{3} \mathrm{~J}=7.2 \mathrm{~Hz},{ }^{3} \mathrm{~J}=2.5 \mathrm{~Hz}, 1 \mathrm{H}$ ), 3.12 (dd, $\left.{ }^{3} \mathrm{~J}=17.3 \mathrm{~Hz},{ }^{3} \mathrm{~J}=2.5 \mathrm{~Hz}, 1 \mathrm{H}\right), 3.62\left(\mathrm{dd},{ }^{3} \mathrm{~J}=17.3 \mathrm{~Hz},{ }^{3} \mathrm{~J}=11.0 \mathrm{~Hz}, 1 \mathrm{H}\right), 3.86\left(\mathrm{dt},{ }^{3} \mathrm{~J}=\right.$ $\left.11.1 \mathrm{~Hz},{ }^{3} \mathrm{~J}=2.5 \mathrm{~Hz}, 1 \mathrm{H}\right), 7.07-7.17$ (m, $\left.1 \mathrm{H}\right), 7.20-7.25$ (m, $\left.4 \mathrm{H}\right), 7.39-7.46$ (m, $\left.2 \mathrm{H}\right)$, 7.59-7.55 (m, $1 \mathrm{H})$, 7.86-7.92 (m, $2 \mathrm{H})$.

${ }^{13}$ C-NMR (90 MHz, $\mathrm{CDCl}_{3}$ ): $\delta 10.7$ (q), 28.4 (q), 34.7 (s), 39.4 (t), 39.9 (d), 49.0 (d), 125.9 (d), 127.4 (d), 128.0 (d), 128.5 (d), 128.6 (d), 137.8 (s), 147.4 (s), 199.3 (s). 


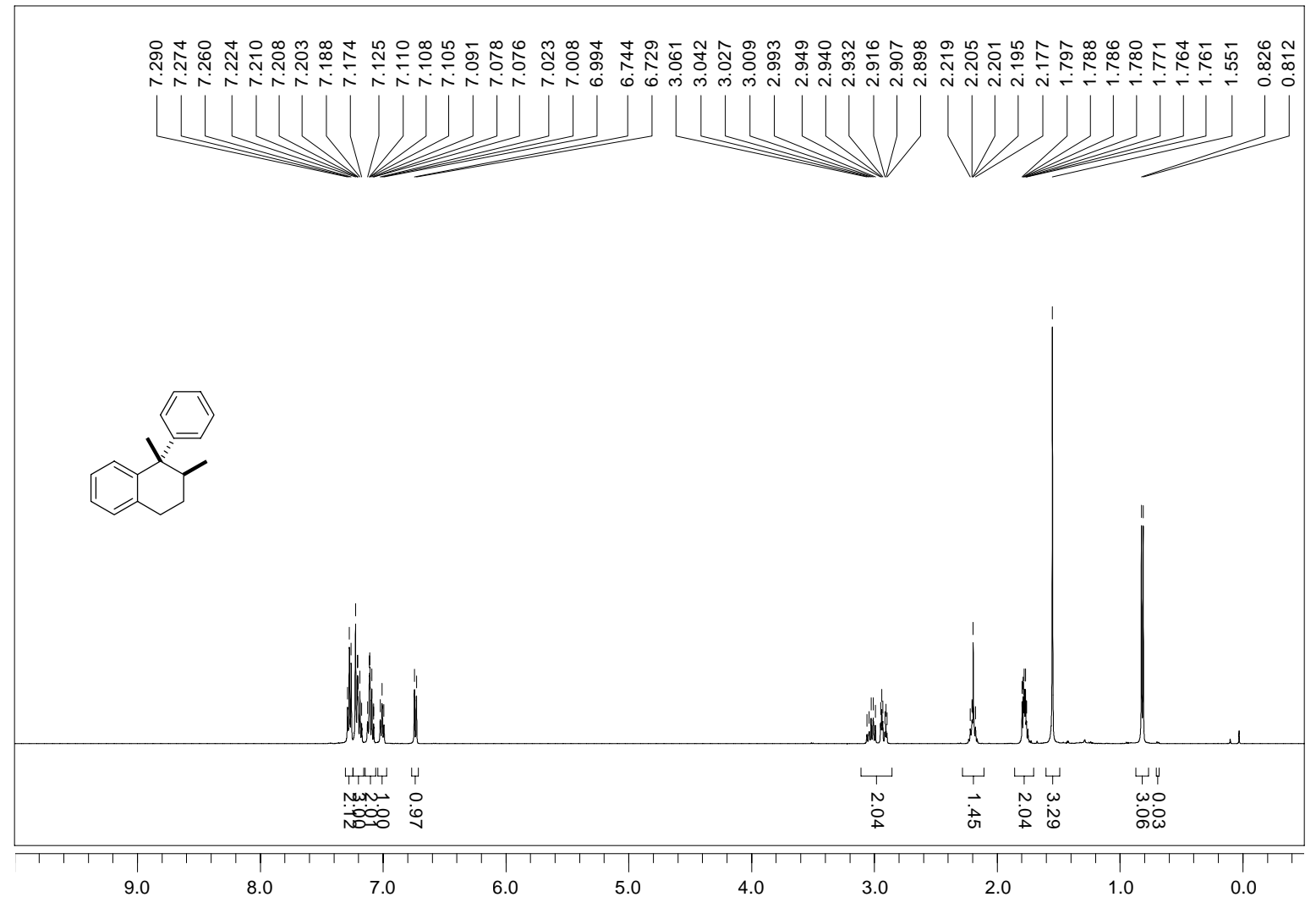

ppm (t1)

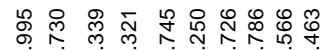

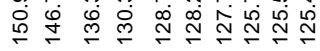
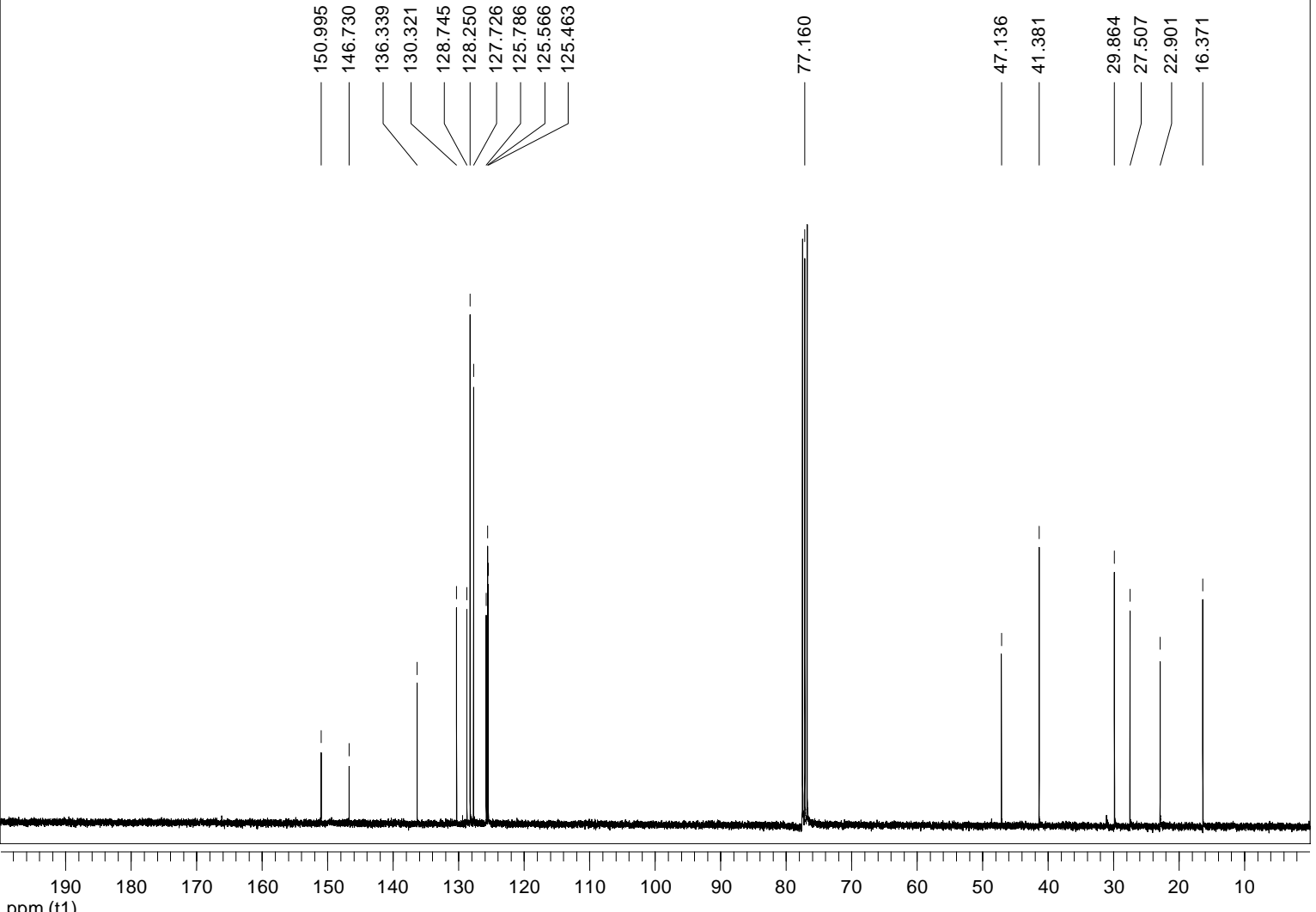
$\operatorname{ppm}(\mathrm{t} 1)$ 


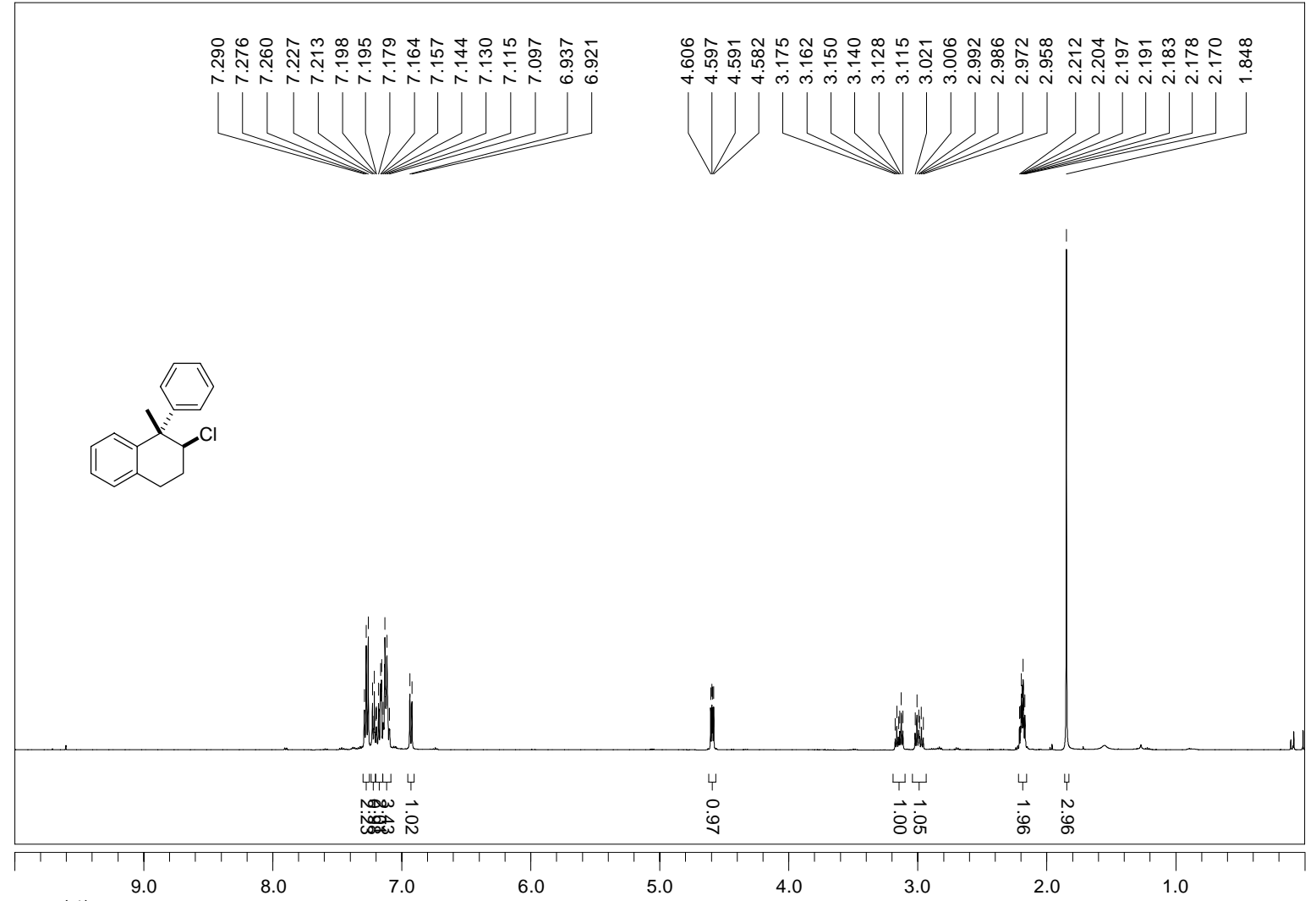

ppm (t1)

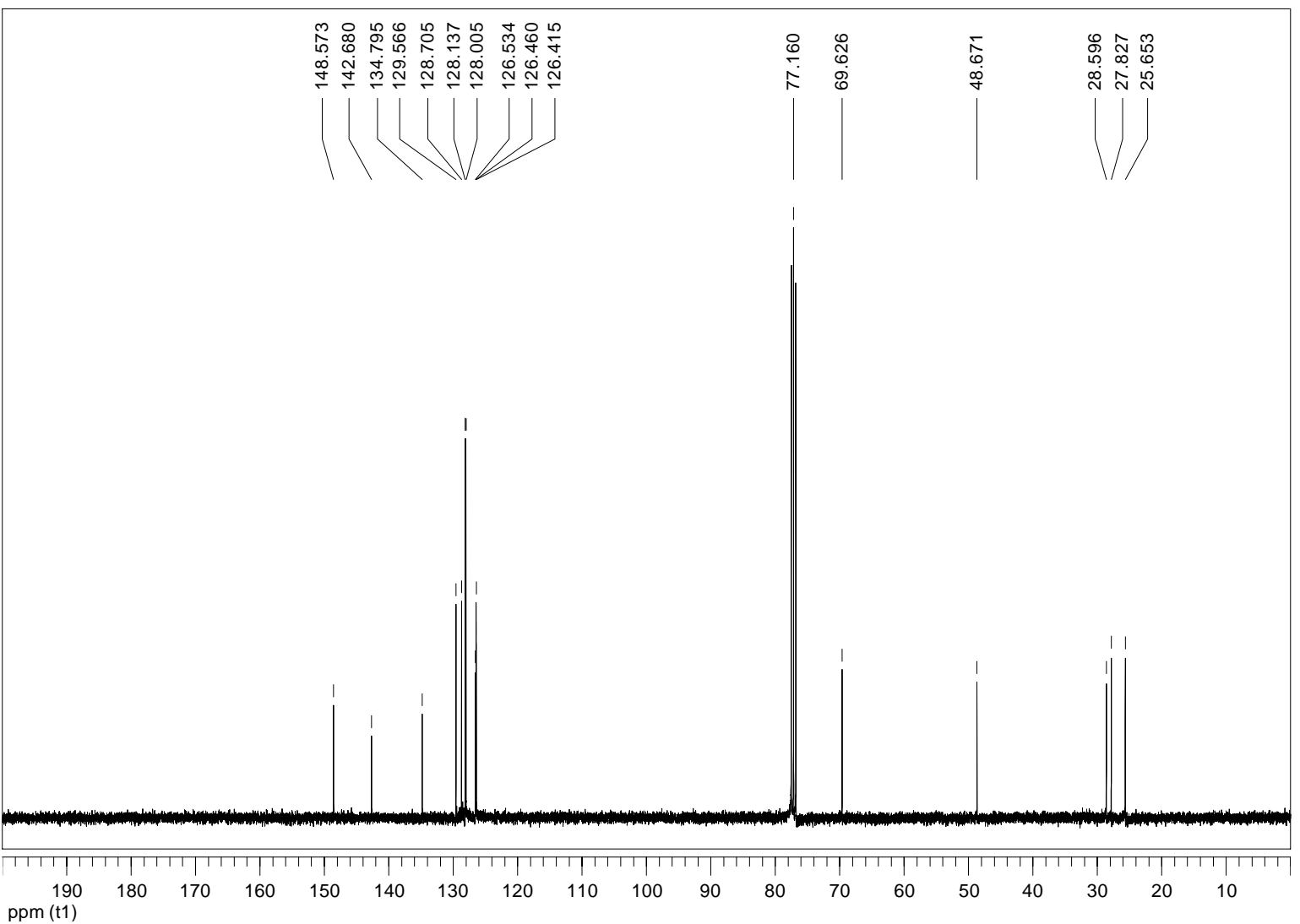




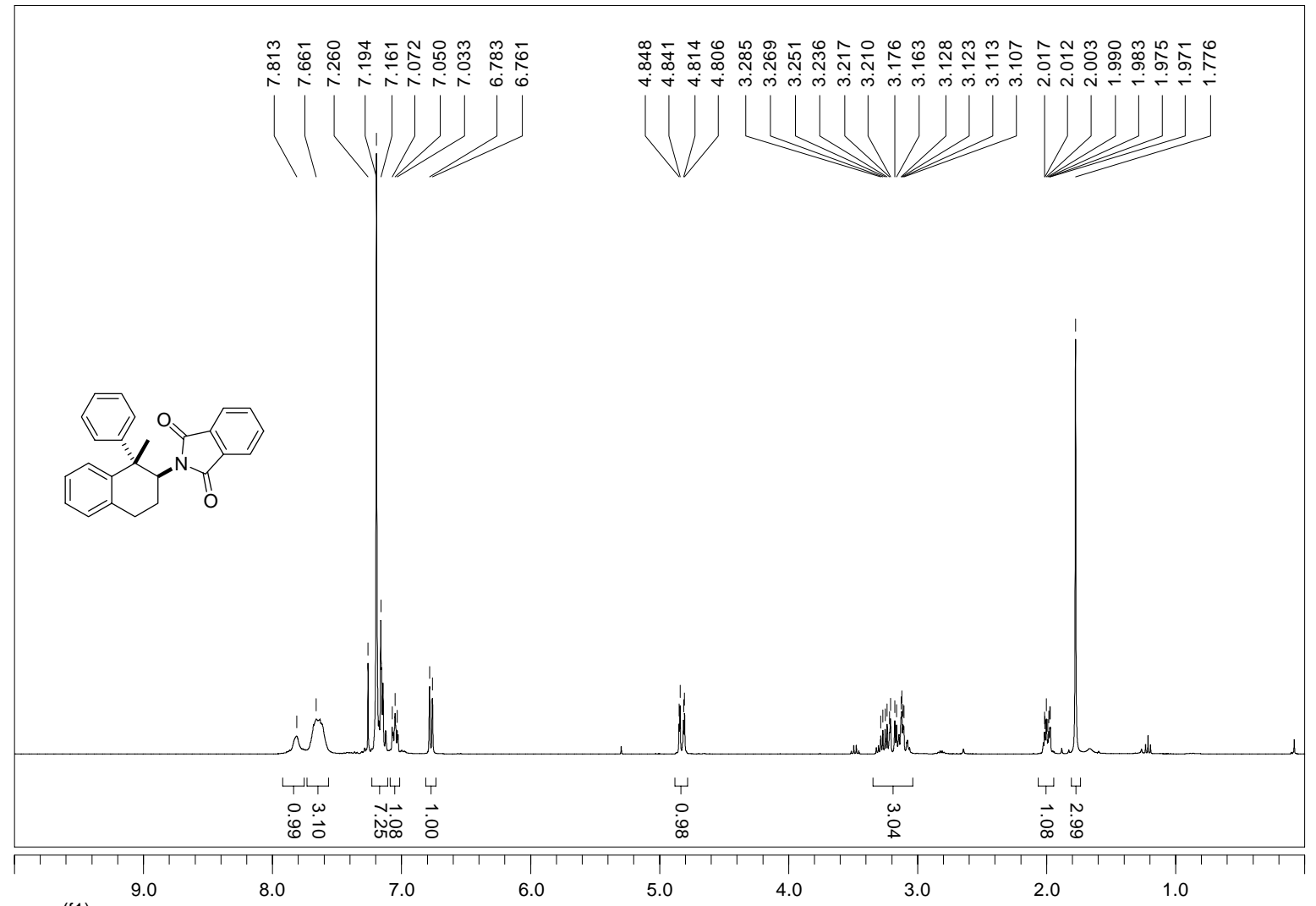

ppm (f1)

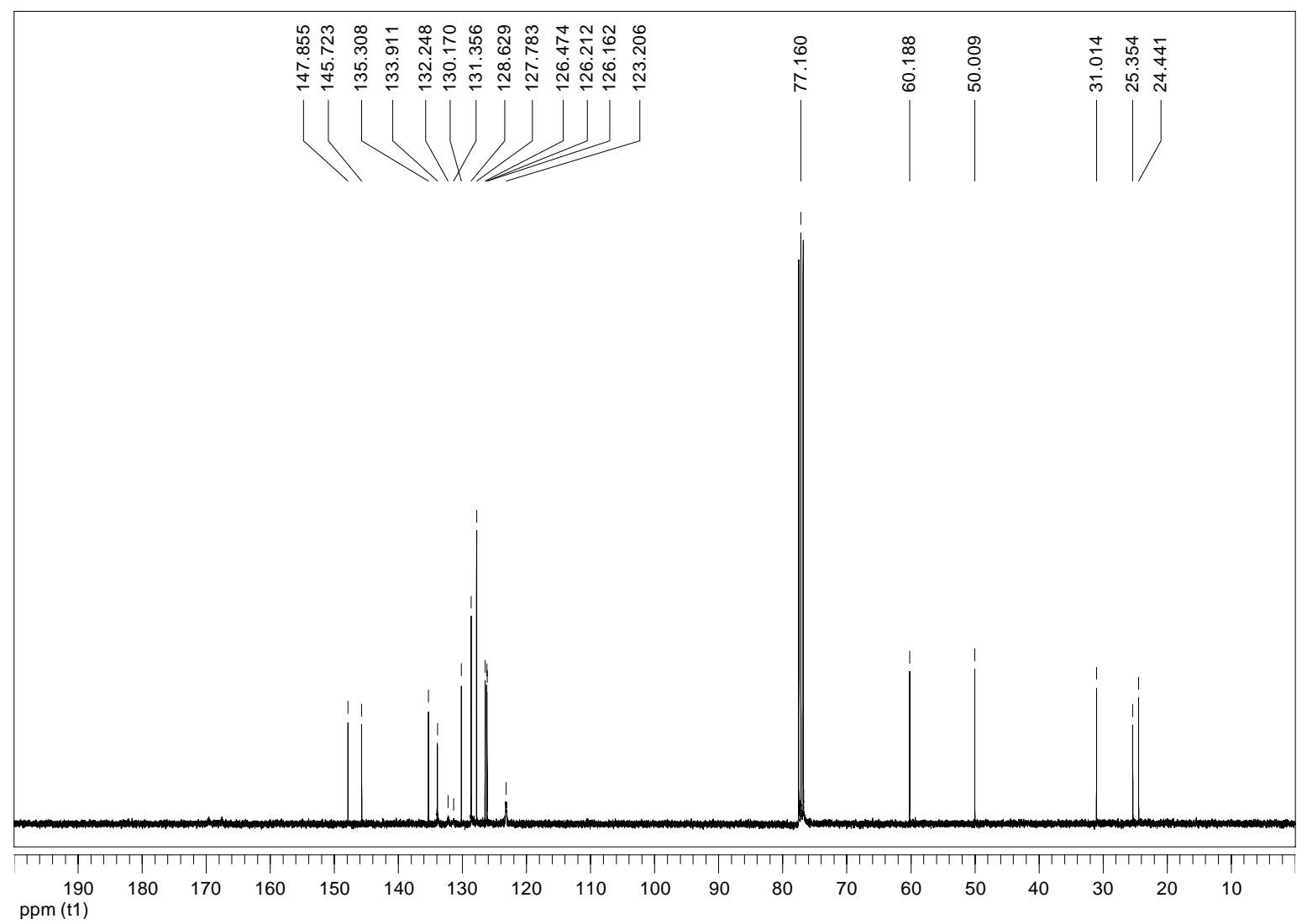




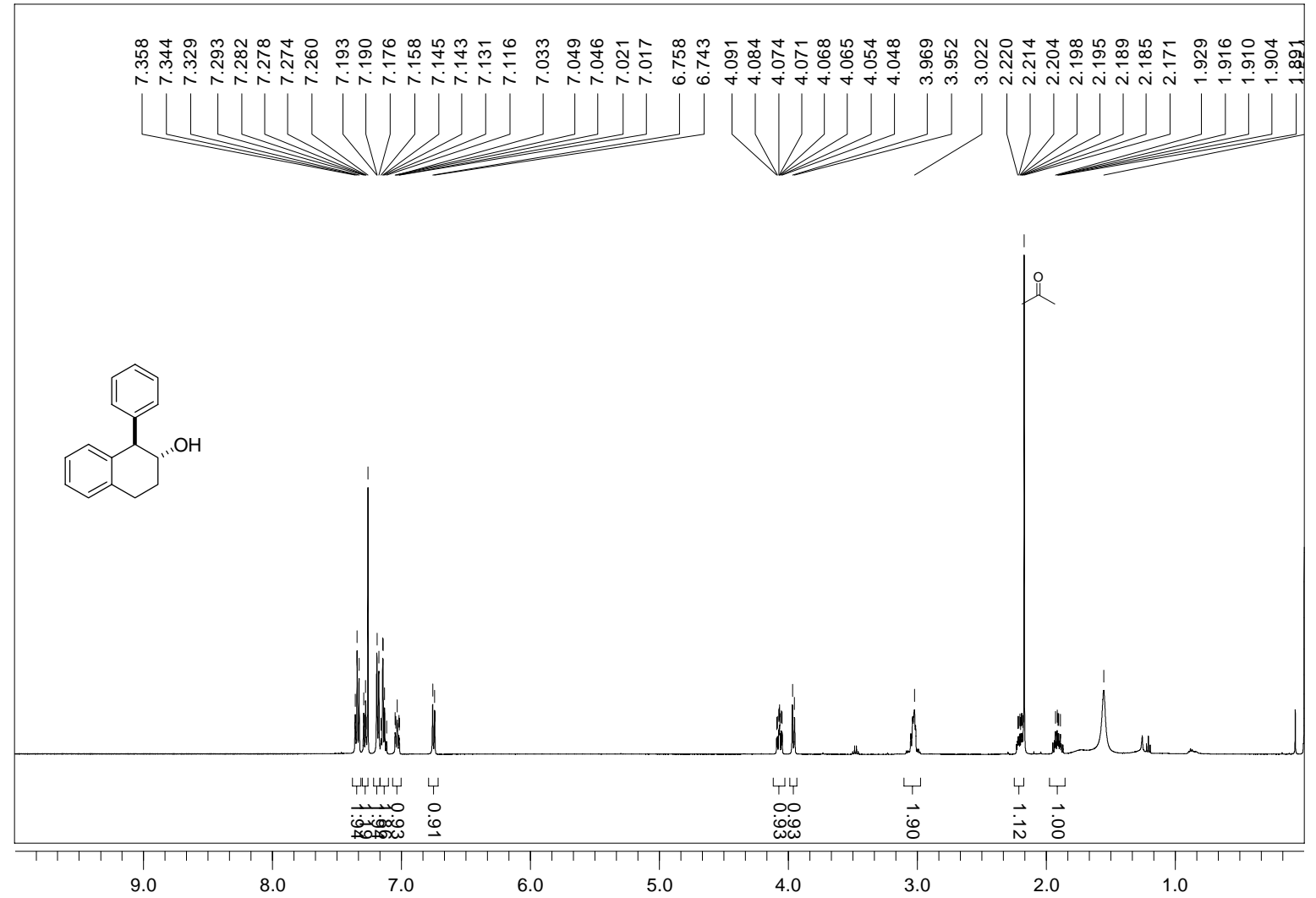

ppm (f1)

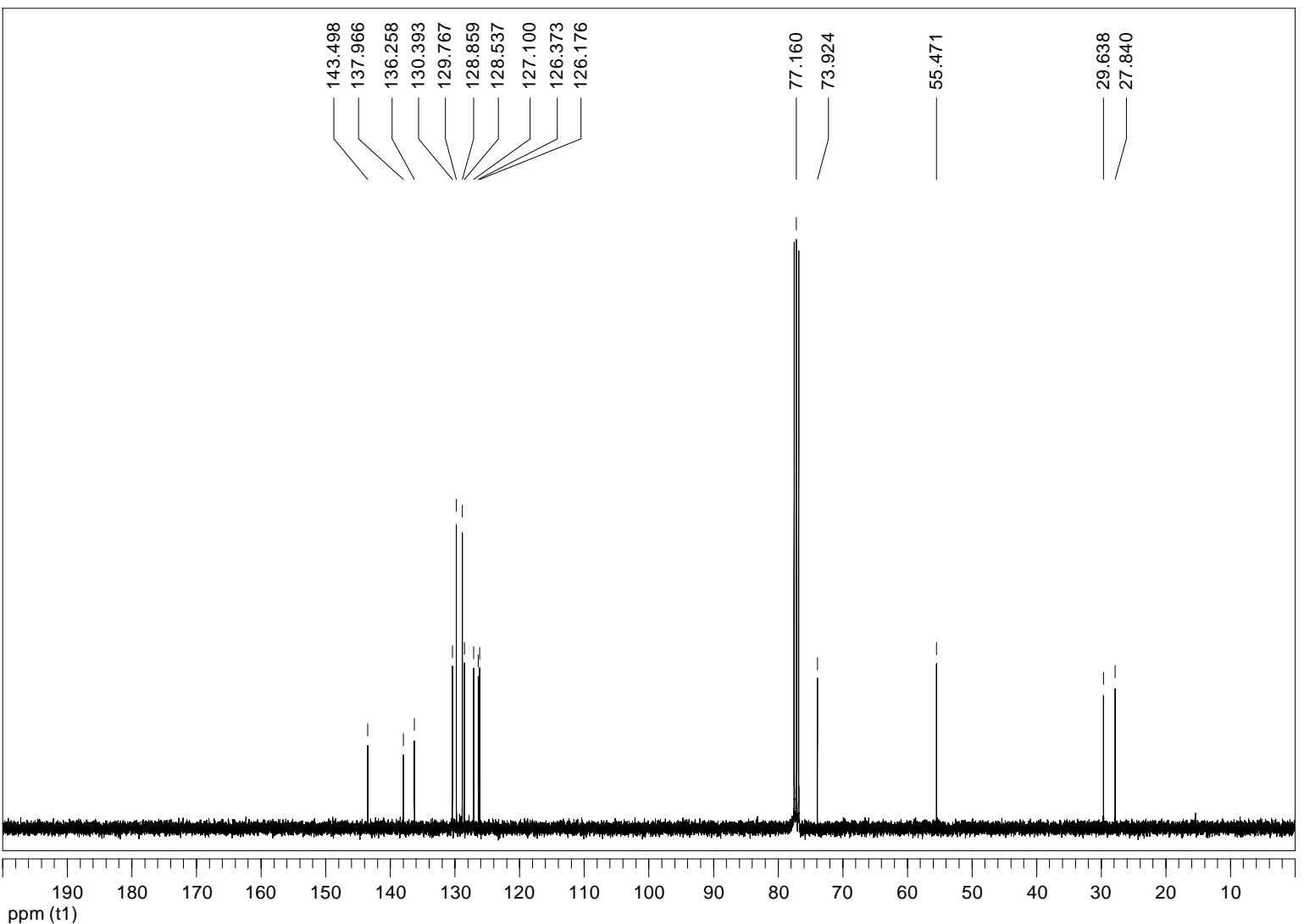




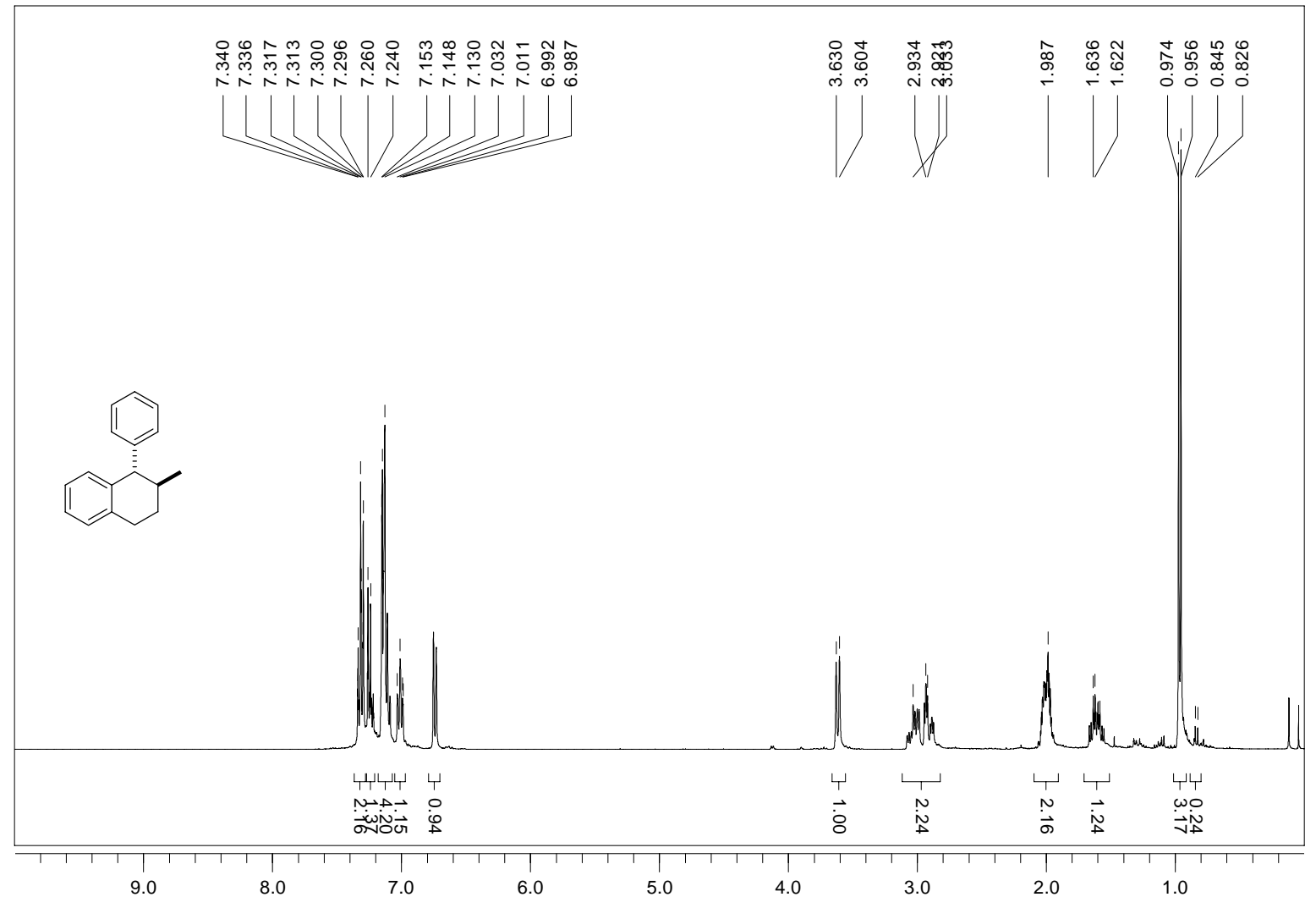

ppm (f1)

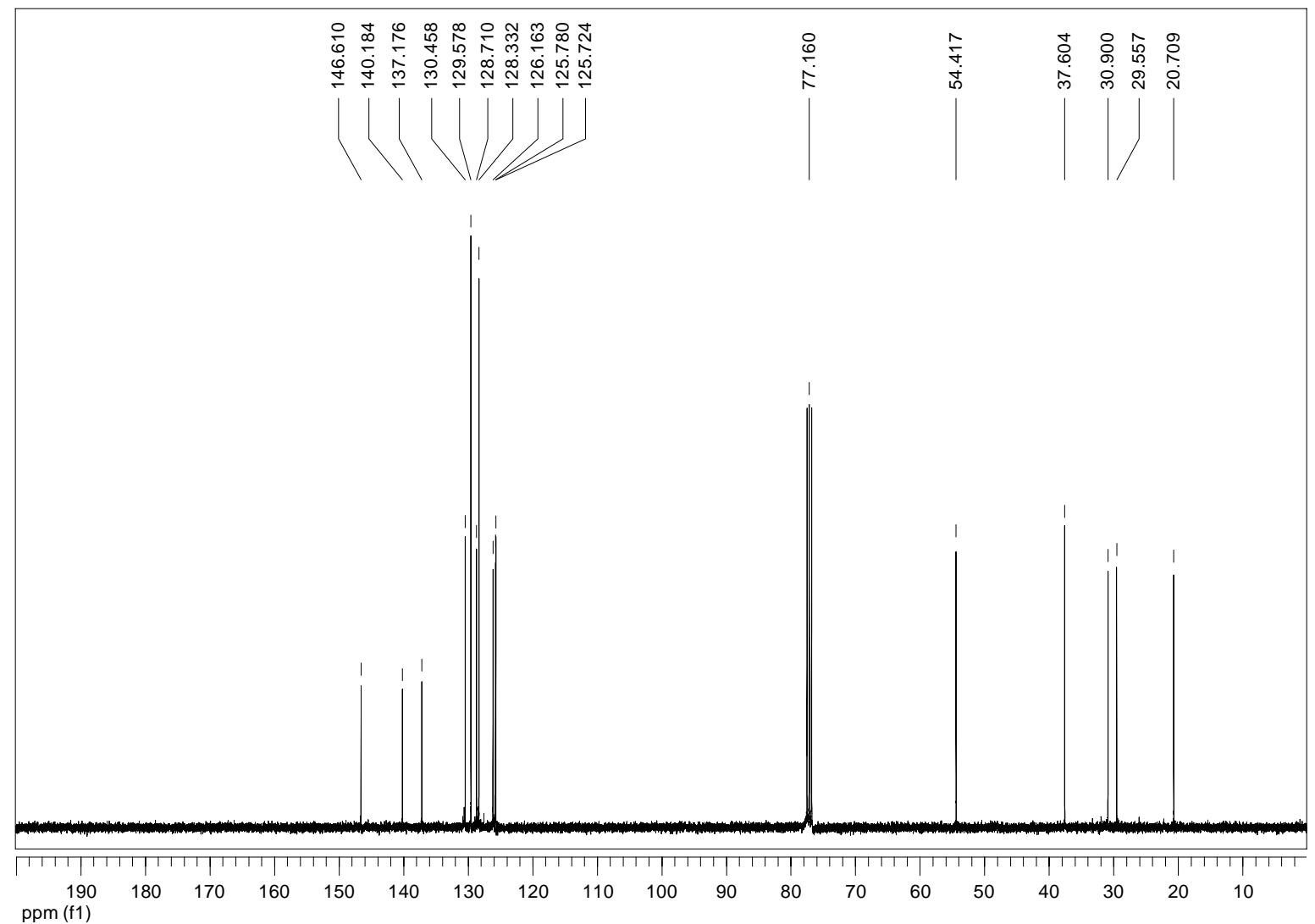




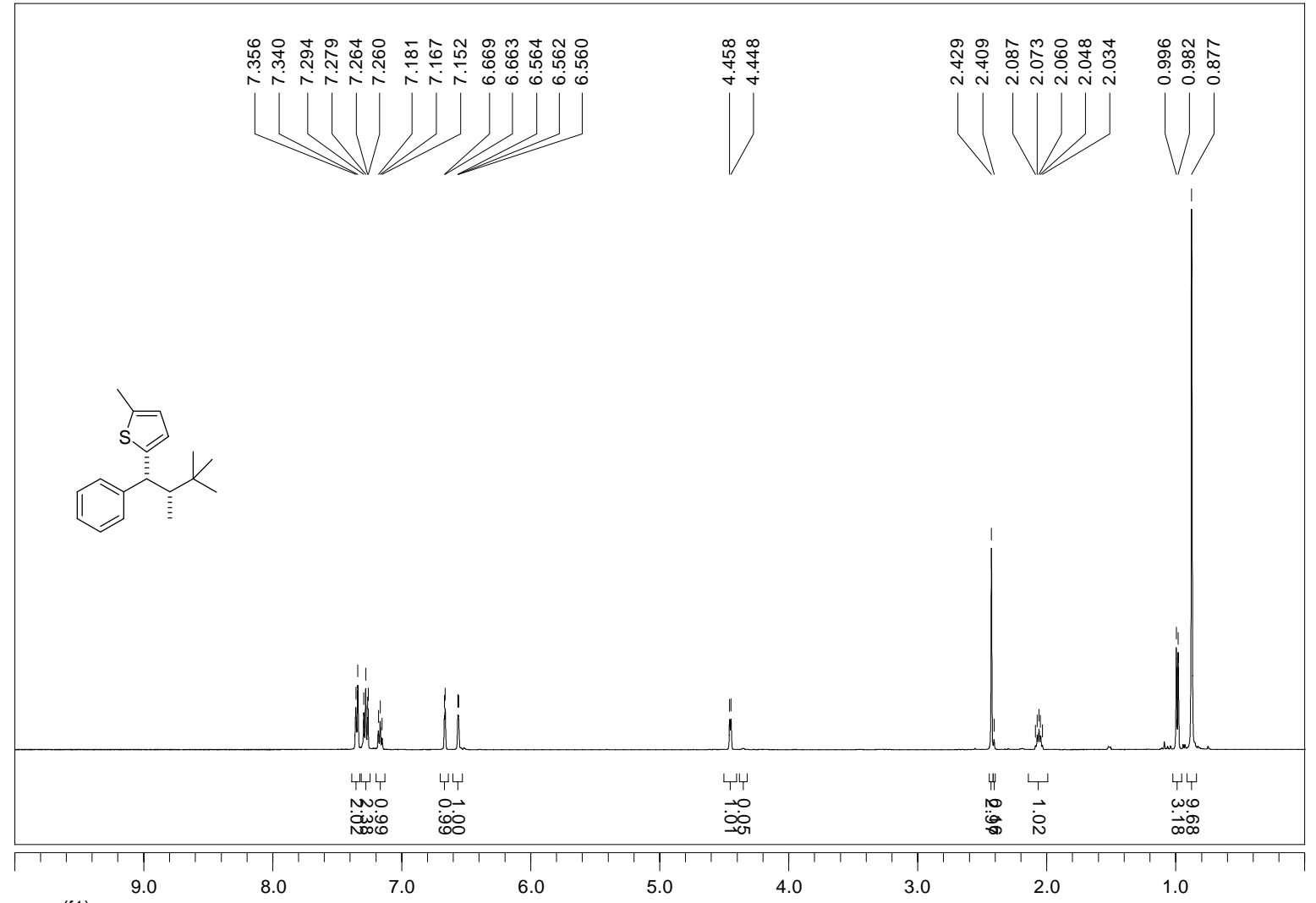

ppm (f1)

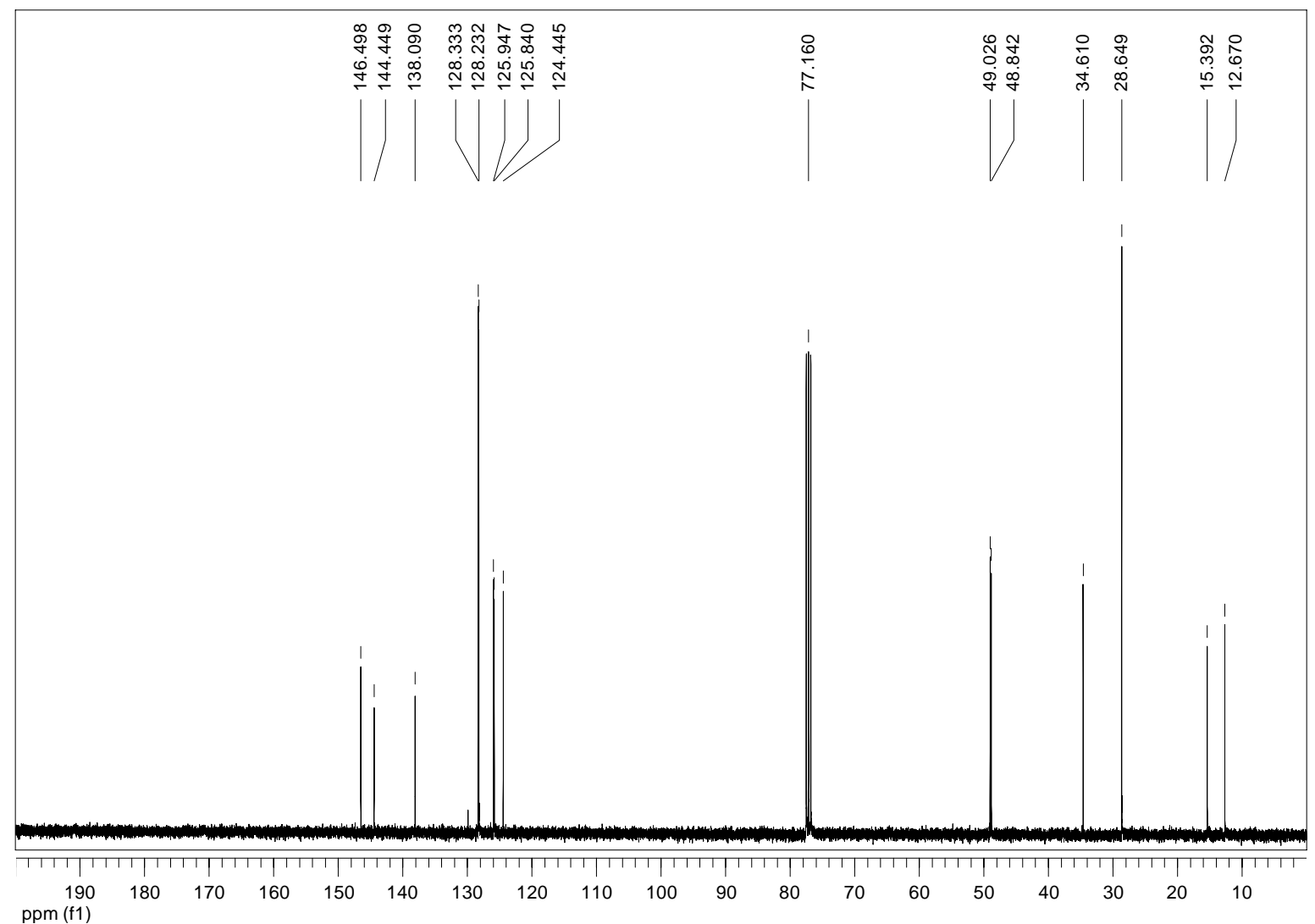




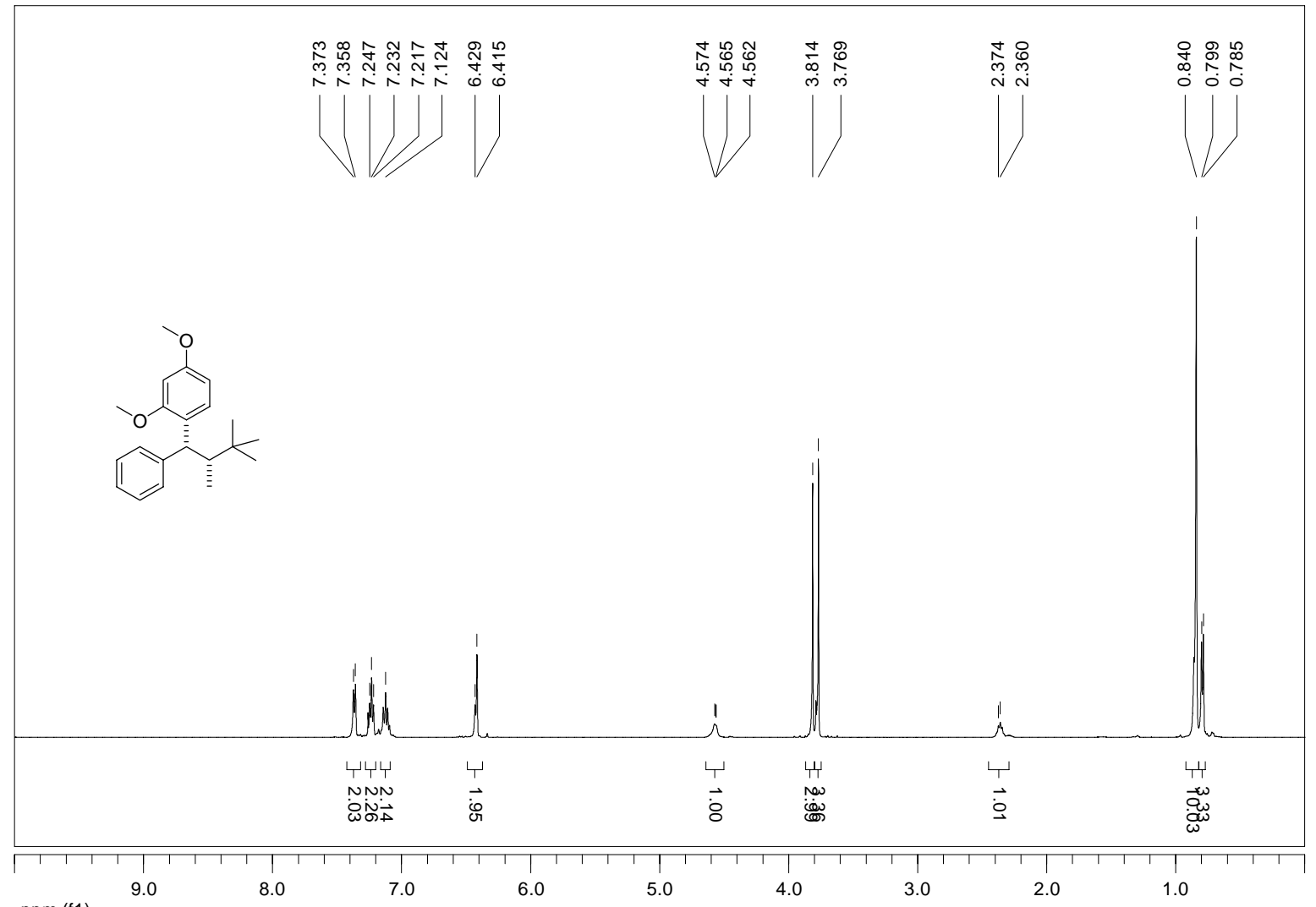

ppm (f1)
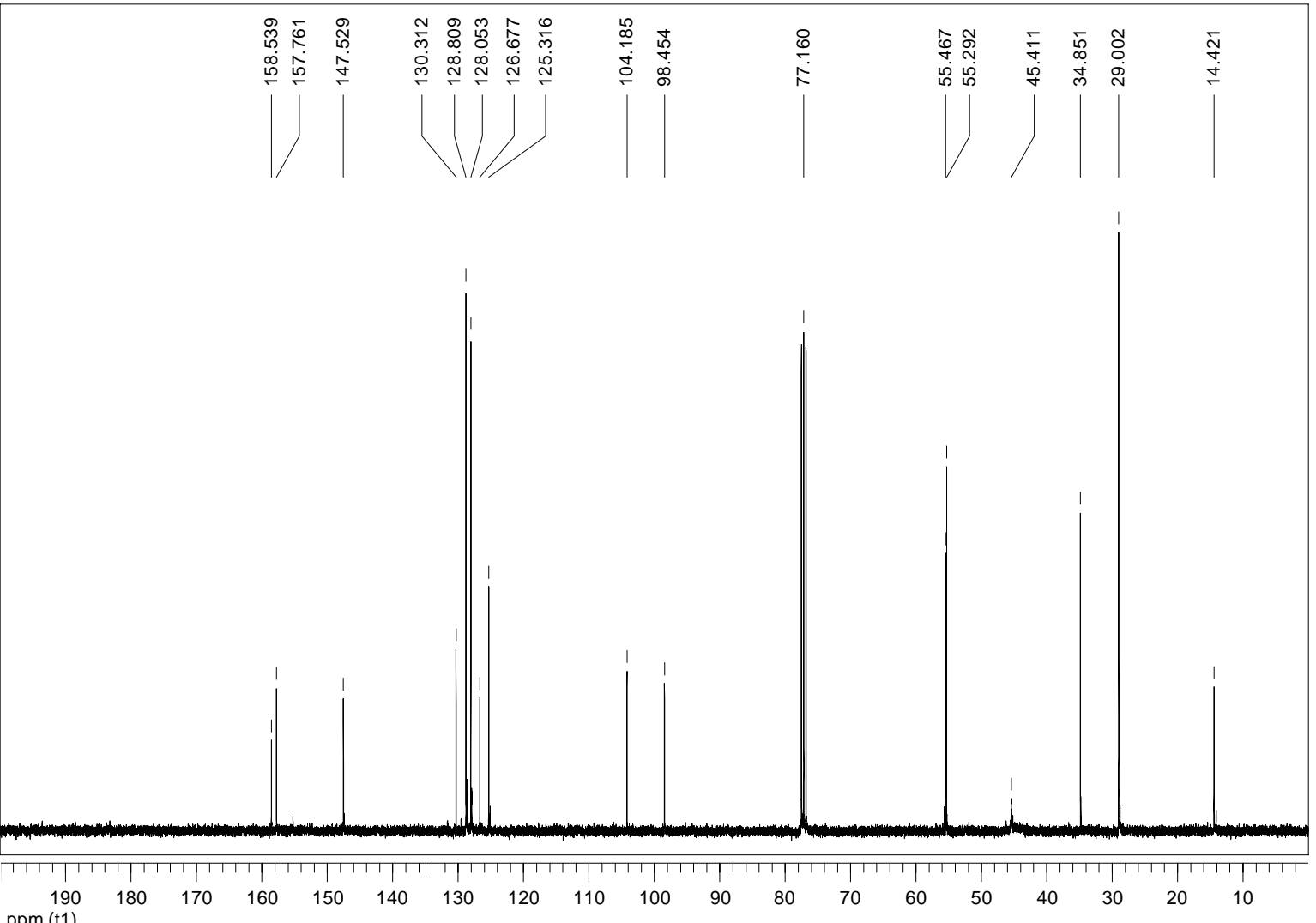
ppm (t1) 


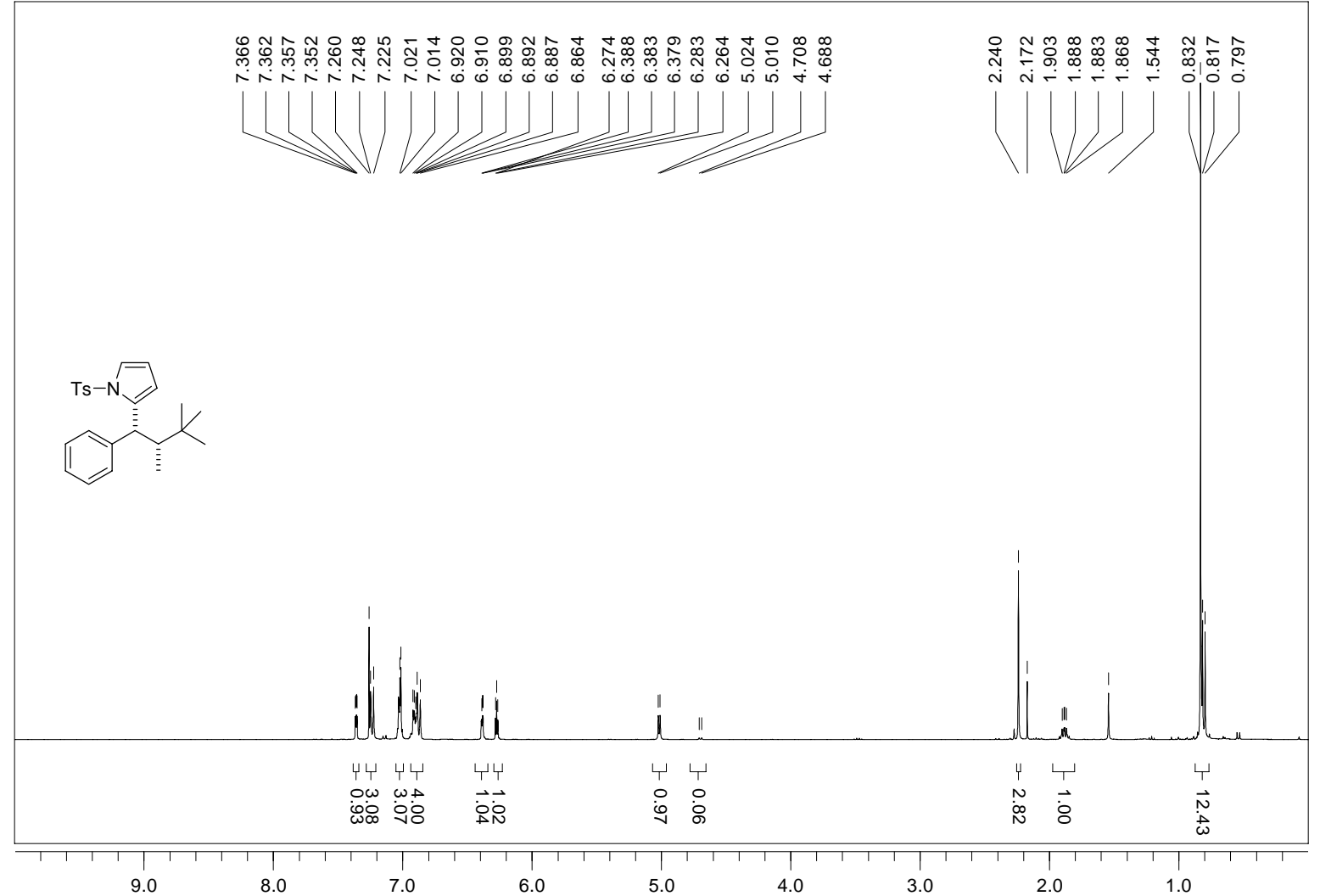

ppm (t1)

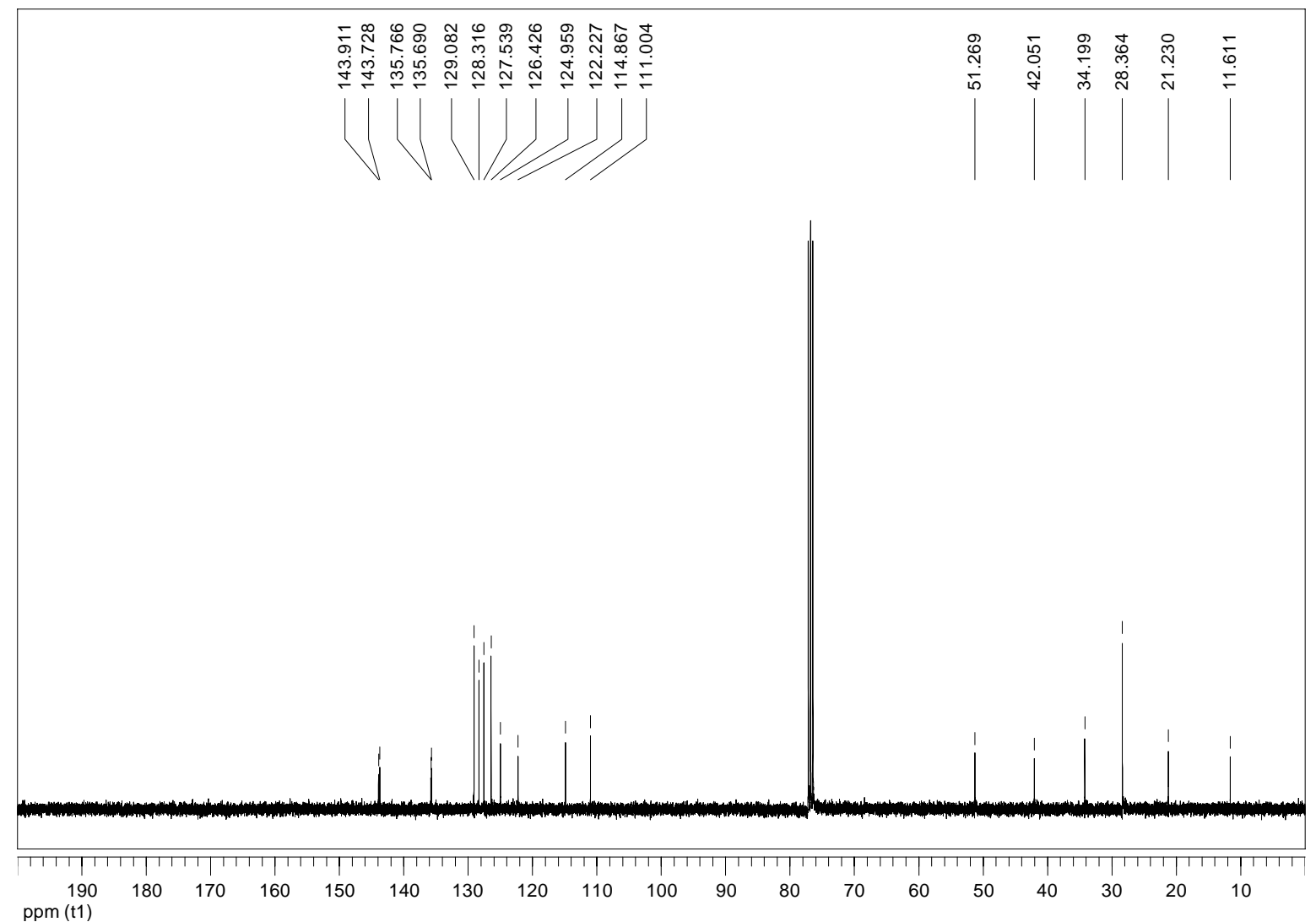




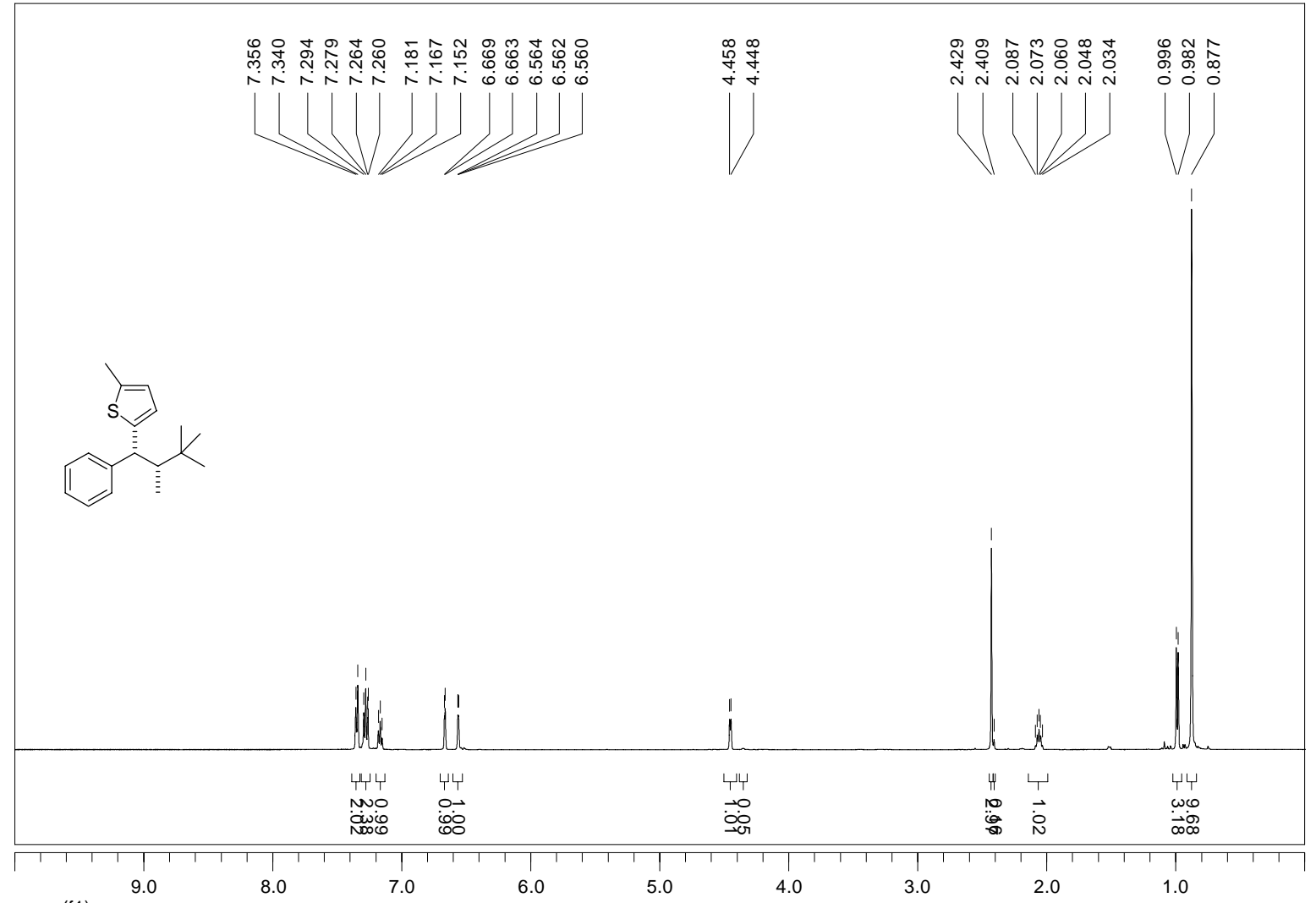

ppm (f1)

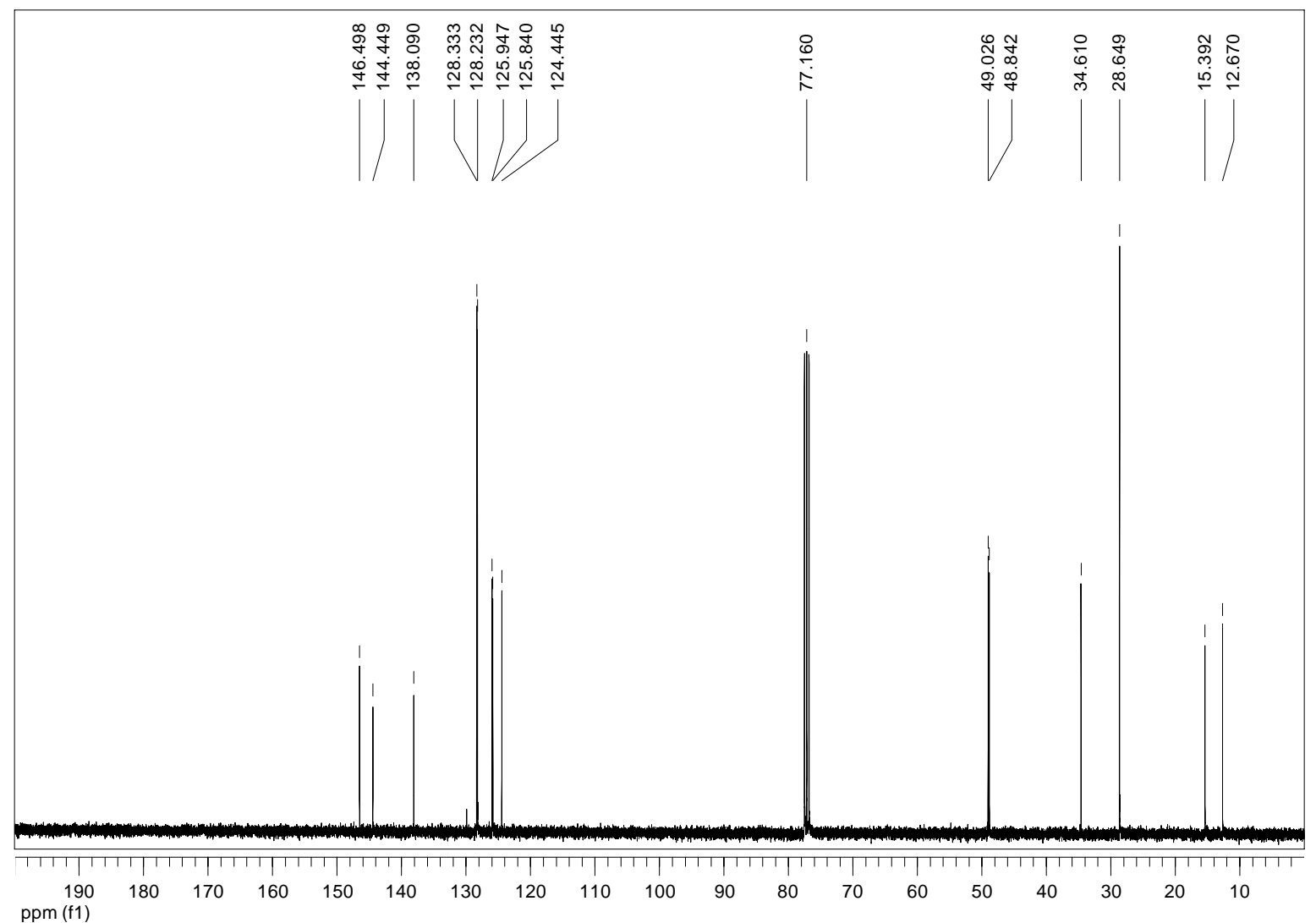




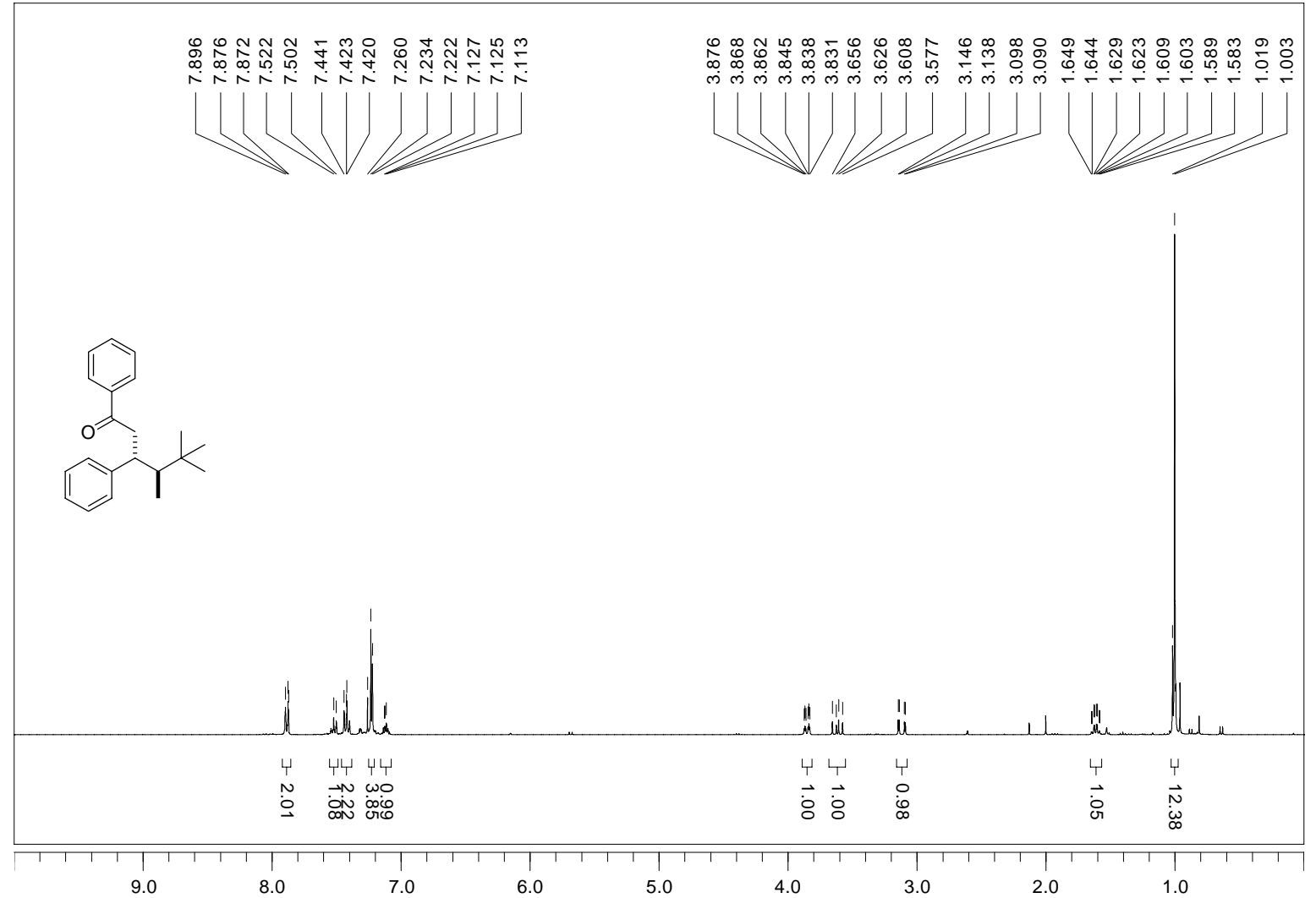

ppm (f1)

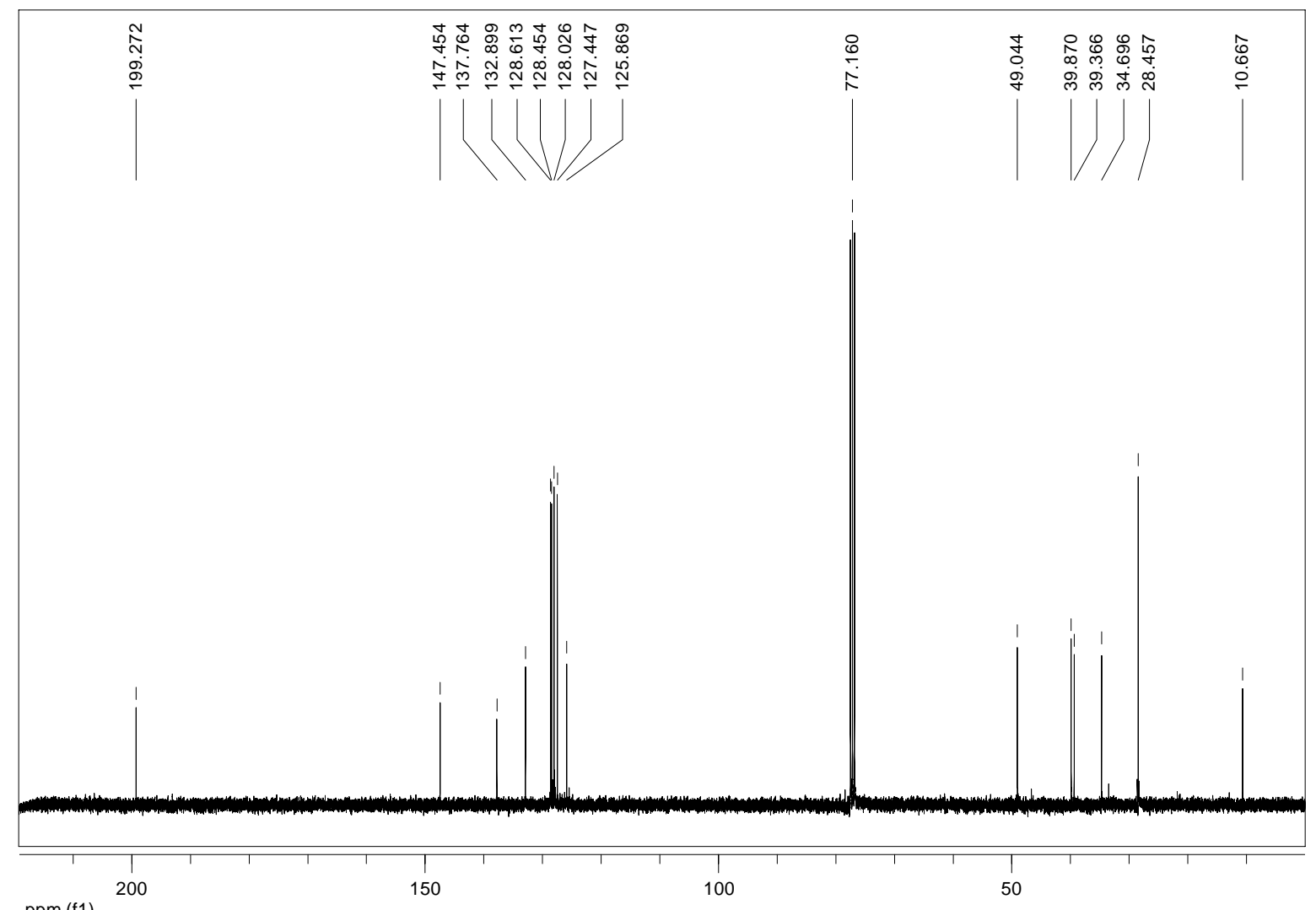

